\title{
Correction: Therapeutic targeting of Id 2 reduces growth of human colorectal carcinoma in the murine liver
}

\author{
M. J. Gray · N. A. Dallas · G. Van Buren - L. Xia - A. D. Yang • R. J. Somcio • P. Gaur • L. S. Mangala • P. E. Vivas-Mejia • \\ F. Fan · A. M. Sanguino - G. E. Gallick · G. Lopez-Berestein · A. K. Sood · L. M. Ellis
}

Published online: 30 April 2020

(c) Springer Nature Limited 2020

\section{Correction to: Oncogene}

https://doi.org/10.1038/onc.2008.356

Following the publication of this Article, it was brought to the attention of the Authors that a control for shRNA knockdown of Id 2 in Fig. 2 was a duplicated and reversed set of bands from another control in the Figure. The row of bands on the western blot for Id3 is the same blot for Id1 knockdown, but "flipped" horizontally. This does not change the conclusions of the manuscript. The authors have submitted a revised Fig. 2, omitting this band, as they no longer have access to the reagents and cannot repeat this part of the experiment.
Fig. 2

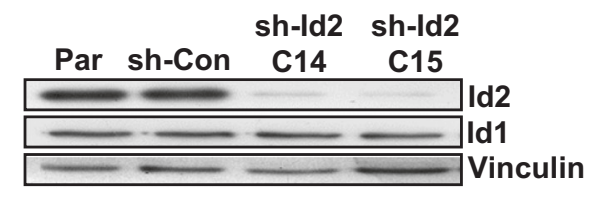

B

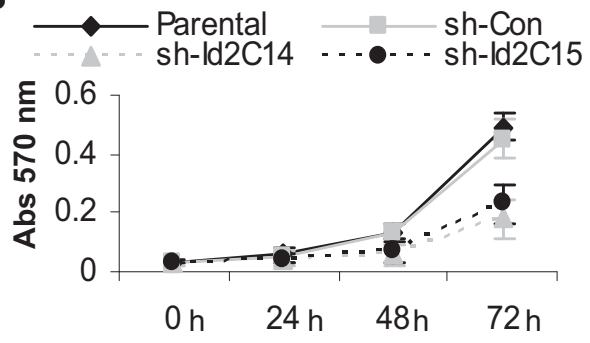

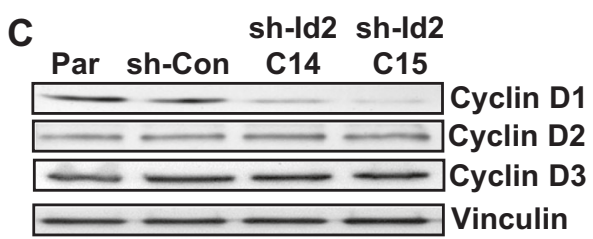

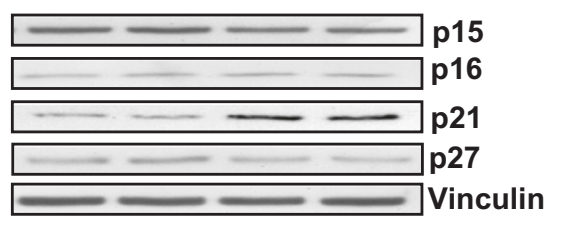

